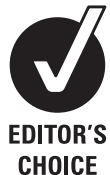

'Department of Pathology, Singapore General Hospital, Singapore, Singapore ${ }^{2}$ Department of Anatomical and Cellular Pathology, Chinese University of Hong Kong, Hong Kong, China

\section{Correspondence to} Dr Puay Hoon Tan, Department of Pathology, Singapore General Hospital, Outram Road, Singapore 169608, Singapore: tan.puay.hoon@sgh.com.sg

Accepted 21 February 2011 Published Online First 12 March 2011

\title{
Histiocytoid breast carcinoma: an enigmatic lobular entity
}

\author{
Puay Hoon Tan, ${ }^{1}$ Oi Harada, ${ }^{1}$ Aye Aye Thike, ${ }^{1}$ Gary Man-Kit Tse ${ }^{2}$
}

\begin{abstract}
Histiocytoid breast carcinoma is an uncommon entity that is mostly regarded as a variant of lobular carcinoma. Its occurrence with apocrine lobular carcinoma in situ and consistent expression of gross cystic disease fluid protein 15 suggest apocrine differentiation. Its recognition is often challenging, particularly when histiocytoid tumour cells occur in a metastatic site before the primary diagnosis of breast carcinoma, or in limited core biopsy or cytology material. In the breast, its bland histological appearances can lead to a benign diagnosis. Clues to the correct conclusion include finding tumour cells with more cytological atypia, the presence of cytoplasmic vacuoles and secretions, coexistence with more traditional invasive lobular carcinoma patterns and/ or lobular neoplasia, and the use of immuohistochemistry to confirm their epithelial nature. Close clinicoradiological correlation and awareness of histological mimics are needed to achieve an accurate diagnosis of this enigmatic condition that should be appropriately subsumed within the invasive lobular histological subtype.
\end{abstract}

Histiocytoid breast carcinoma was first described by Hood et al $^{1}$ in 1973, in which 13 cases of tumour metastatic to the eyelid were documented, and eight of these metastases featured histiocytoid appearances that caused diagnostic challenges. It has since been variously ascribed to lobular or apocrine carcinoma, ${ }^{2-9}$ as well as being linked to lipid-rich carcinoma, ${ }^{1}{ }^{10}$ with some authors advocating that it be considered a distinct entity. ${ }^{11}$

Despite the several small series and individual case reports that have been presented in the literature, $^{26-9} 11-15$ there is still no universal agreement on how this enigmatic tumour ought to be specifically classified, or whether its recognition has prognostic or predictive significance. ${ }^{16} 17$ Its mimicry of benign and other conditions, however, is well recognised, and remains a pitfall for histopathologists, especially when assessing limited material on core biopsy or cytology.

In this review, we appraise the information on histiocytoid breast carcinoma since its initial description and discuss differential diagnoses that may pose interpretive issues.

\section{BACKGROUND AND HISTORY}

Although the first documentation of histiocytoid breast carcinoma by Hood et a ${ }^{1}$ was formally published in 1973, the authors alluded to having recognised this 'peculiar variant' of metastatic breast carcinoma for several years. Their 13 cases had histological confirmation of both primary breast tumours and metastases to the eyelid, with five cases presenting clinically with the eyelid lesion before discovery of the breast tumour, and in which initial erroneous histological diagnoses of the eyelid lesion included xanthelasma, chronic inflammation and benign xanthoma. In a report of 13 cases of lipid-rich carcinoma of the breast that followed soon after, Ramos and Taylor ${ }^{10}$ mentioned the tendency for lymph node metastases to mimic 'some form of histiocytosis', and implied that histiocytoid carcinomas in the paper by Hood et al ${ }^{1}$ were synonymous with lipid-rich cancers, although Hood et $a l^{1}$ had explicitly stated that fat stains were negative in their three cases that were tested, with mucin detected in tumour cell cytoplasm instead, contrasting with the presence of neutral lipid in four cases assessed in the series of Ramos and Taylor. ${ }^{10}$ Neutral fat was also demonstrated in a case report of lipid-rich carcinoma, ${ }^{18}$ indicating its difference from histiocytoid breast carcinoma.

Eusebi et $a l^{\beta}$ noted the association of histiocytoid invasive breast carcinoma with apocrine lobular carcinoma in situ, and concluded that histiocytoid tumour cells manifested apocrine differentiation based on their immunohistochemical reactivity with gross cystic disease fluid protein 15 (GCDFP-15). The authors later described a series of 10 aggressive pleomorphic invasive lobular cancers of which five included histiocytoid cells with granular foamy cytoplasm, ${ }^{5}$ and commented that apocrine differentiation in lobular carcinoma could assume typical apocrine, histiocytoid and pleomorphic appearances. In another paper documenting 13 histiocytoid breast cancers that were also termed 'myoblastomatoid', they demonstrated apocrine differentiation in all using immunohistochemistry and in-situ hybridisation, and cautioned about the potential misdiagnoses of these cases as benign conditions. Precise prognostic judgements could not be made for this group of histiocytoid breast carcinomas. Kasashima et $a l^{19}$ concurred with the controversial prognosis of lobular carcinoma with histiocytoid features, but found more frequent lymph node metastasis, shorter disease-free survival and higher mortality in their eight cases when compared with 14 age and tumour size-matched examples of classic invasive lobular cancer. They suggested that the expression of MUC2 and MUC5AC in histiocytoid tumours augured a worse outcome.

Gupta et $a l^{20}$ reported a series of 11 histiocytoid breast tumours, all of which had an invasive lobular pattern, with eight cases showing accompanying lobular carcinoma in situ. E-cadherin immunohistochemistry was negative in eight cases, but disclosed moderate to strong membrane reactivity in the remaining three tumours, although the figure 
depicted in their paper showed incomplete membrane staining in some cells suggesting aberrant E-cadherin expression. GCDFP-15 was present in all cases. Based on the presumed ductal differentiation with positive E-cadherin staining in three of their cases, the authors concluded that histiocytoid breast carcinoma did not belong to a specific phenotype and that both lobular and ductal origins were possible.

\section{PATHOLOGICAL FINDINGS Light microscopy}

These tumours consist of sheets of pale histiocyte-like cells with ample, delicate, sometimes finely vacuolated to granular cytoplasm and inconspicuous cell membranes percolating the breast parenchyma (figure 1A). A 'ground-glass' appearance has been used to describe the cytoplasm. ${ }^{1}$ Nuclei are dark to vesicular and are centrally as well as eccentrically placed. Small nucleoli can be discernible. Mitoses are generally scarce. Nuclear pleomorphism is mild to moderate, with mostly grade 1 to 2 nuclei described, ${ }^{20} 21$ despite the incorporation of histiocytoid features in conjunction with pleomorphic lobular breast cancer. ${ }^{5}$ Rare cytoplasmic vacuoles can be discerned (figure 1B). The cells are disposed individually and in loose aggregates, without obvious glandular differentiation, and occasionally stream as linear arrays around resident benign lobules in a 'targetoid' arrangement $^{1-3} 69111522$ (figure 1C). Accompanying lobular neoplasia (atypical lobular hyperplasia or lobular carcinoma in situ) may be identified (figure 1D), which may feature histiocytoid cells or those of conventional or apocrine lobular phenotype.

\section{Histochemistry}

The majority of reports have demonstrated cytoplasmic mucin with Alcian blue and/or periodic acid Schiff stains $\begin{array}{lllll}3 & 5 & 6 & 15 & 22\end{array}$ (figure 2A). Eusebi et $a l^{3}$ referred to the positive cytoplasmic reactivity as 'intracytoplasmic blue halos', condensed blue crescent-like staining, or red granules, respectively. ${ }^{2}$ When performed, lipid stains have been mostly negative, ${ }^{1} \quad 2122$ although one report mentioned the presence of fine fat droplets in a few histiocytoid cells. ${ }^{15}$ Grimelius stains are negative. ${ }^{3}$

\section{Immunohistochemistry}

Immunohistochemistry reveals positive reactivity of the histiocyte-like cells for epithelial markers such as AE1/3, MNF116, Cam 5.2, CK7, EMA confirming an epithelial origin ${ }^{2} 422$ (figure $2 \mathrm{~B}$ ). Strong positivity for CEA has also been reported. ${ }^{11}$ S100 is generally negative, ${ }^{21}$ with Eusebi et al ${ }^{4}$ mentioning weak staining in one of 13 histiocytoid carcinomas of the breast in their series.

E-cadherin is predominantly negative (figure 2C). ${ }^{6}{ }^{20}$ Gupta et $a l^{20}$ described three positively stained cases, leading to their conclusion that histiocytoid breast carcinoma lacked a distinct immunophenotype, and could therefore belong to either lobular or ductal subtypes. Their E-cadherin-positive case illustration, however, showed a few cells with apparent incomplete membrane staining, which may reflect aberrant protein expression by a dysfunctional E-cadherin gene. ${ }^{23}$ Rakha et al ${ }^{24}$ also recently described E-cadherin expression in $16 \%$ of 239 cases of histologically confirmed invasive lobular carcinomas, of which the majority were associated with abnormal expression of one or more members of the E-cadherin-catenin complex, implicating a lack of functionality of the E-cadherin gene.

GCDFP-15 is consistently positive in studies in which it was applied $^{3-5} 2022$ (figure 2D), and this has been interpreted as evidence of apocrine differentiation. ${ }^{3} 25-27$ Oestrogen and progesterone receptor expression and cerbB2 status are variable. $^{19} 22$

Kasashima et $a l^{19}$ extended their investigation into mucin profiles of eight histiocytoid and 14 classic invasive lobular carcinomas using immunohistochemistry. Histiocytoid lobular cancers showed positive reactivity for MUC2 and MUC5AC in $75 \%$ and $50 \%$ of cases, respectively, while almost all classic forms were negative. Both histiocytoid and classic lobular carcinomas were positive for MUC1 and negative for MUC4 and MUC6. It was postulated that the expression of non-mammary
Figure 1 (A) Invasive histiocytoid carcinoma consists of sheets of pale tumour cells with ample cytoplasm with dark nuclei that are sometimes eccentrically placed. (B) Signet ring cells and cells with cytoplasmic vacuoles containing luminal secretions are present. (C) Pale histiocytoid cells streaming around a lobule in a targetoid fashion. (D) Atypical lobular hyperplasia is present.
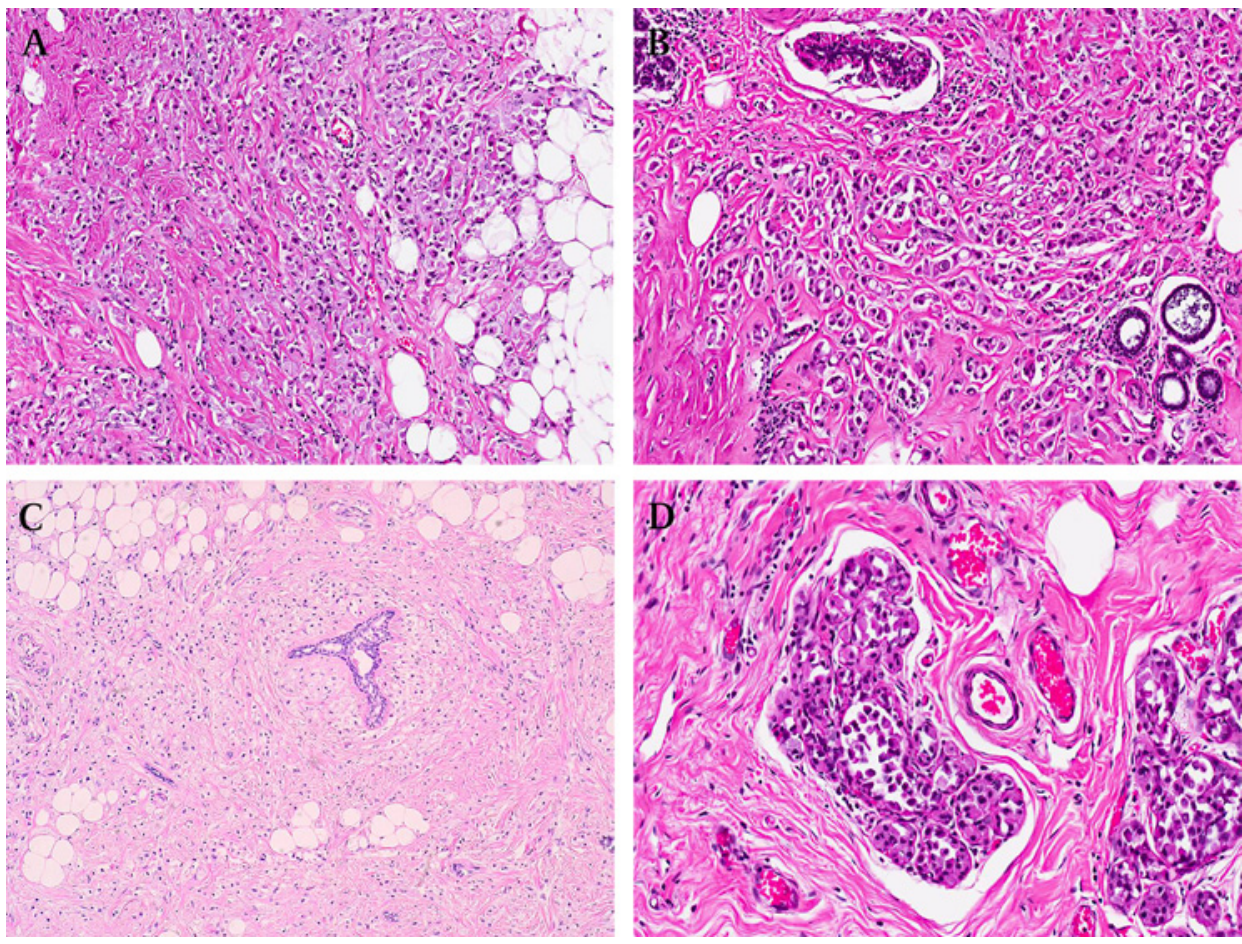
Figure 2 (A) Mucicarmine stain shows pink cytoplasmic secretions. (B) Immunohistochemistry for the epithelial marker AE1/3 shows diffuse positive reactivity in the histiocytoid tumour cells. (C) E-cadherin is negative in the tumour cells, while benign ductular epithelium and myoepithelial cells are positive. Lobular neoplastic cells of atypical lobular hyperplasia are also negative. (D) Diffuse positivity for GCDFP-15 immunohistochemistry.
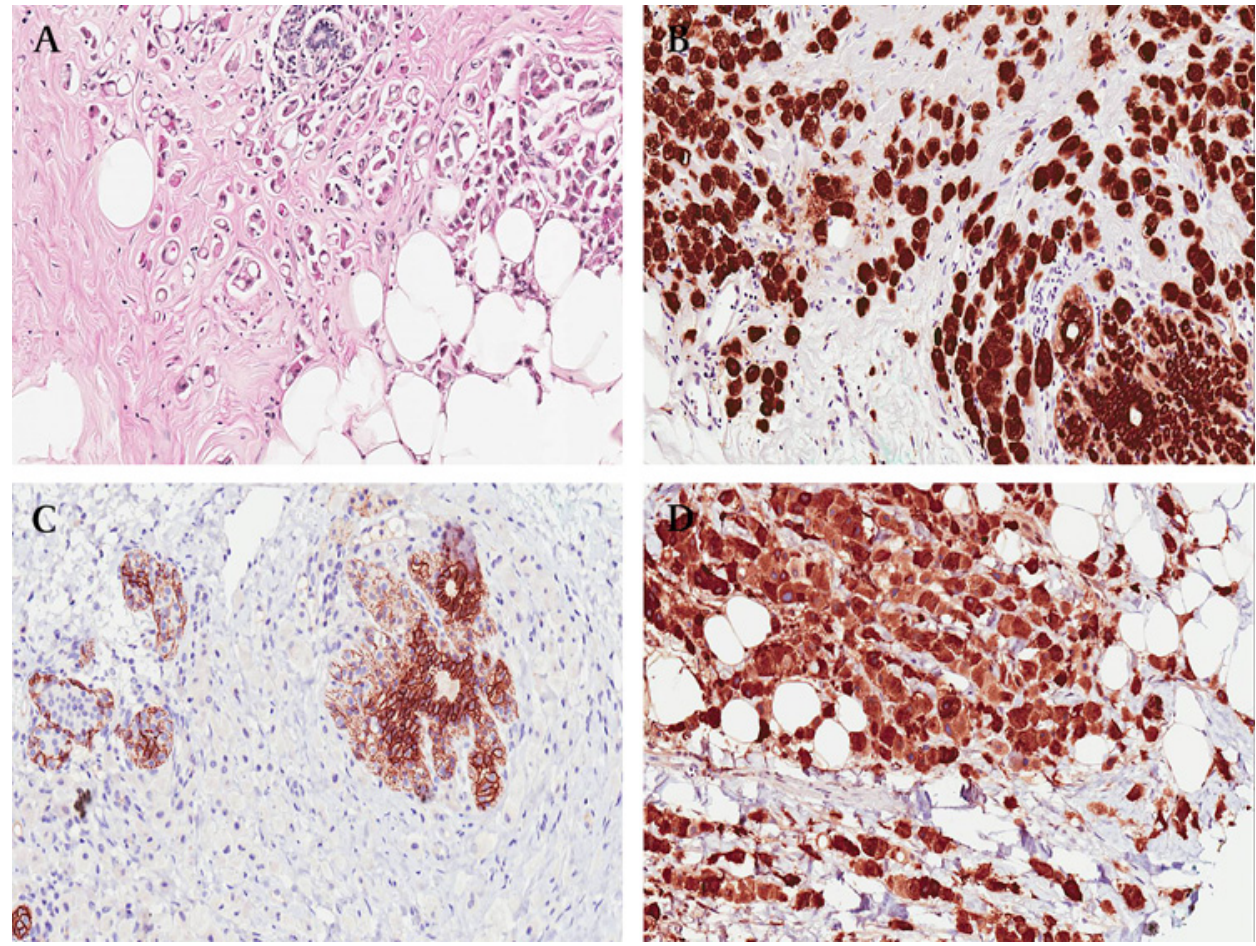

mucins (MUC2 and MUC5AC) could account for the worse prognosis of histiocytoid carcinomas in their study.

\section{Electron microscopy}

Ultrastructural studies show abundant cytoplasm with poorly developed organelles and small numbers of mitochondria, several lysosomes and Golgi apparatus. Mitochondria can be large with incomplete cristae. ${ }^{3}$ Membrane-bound electron-dense granules ranging from 300 to $800 \mathrm{~nm}^{22}$ and from 166 to $320 \mathrm{~nm}^{3}$ are present. Nuclei are round to oval with small nucleoli, thin nuclear membranes and fine chromatin. Intercellular junctions are rudimentary. ${ }^{15} 22$

\section{Molecular studies}

There are scant specific molecular data on histiocytoid breast carcinoma. Extrapolating from its presumed apocrine differentiation due to uniform GCDFP-15 expression, apocrine invasive lobular cancer has been shown to harbour molecular overlap with the pleomorphic variant, ${ }^{28} 29$ with both tumours demonstrating genetic similarity to classic invasive lobular tumours on expression profiling. In a recent detailed study on 31 in-situ pleomorphic lobular breast carcinomas, of which 13 were of apocrine cytomorphology, Chen et al ${ }^{30}$ discovered that pleomorphic lobular carcinoma in situ demonstrated $16 \mathrm{q}$ loss and $1 \mathrm{q}$ gain similar to the classic variety, with apocrine forms displaying significantly more alterations.

\section{DIFFERENTIAL DIAGNOSES}

The relatively bland cytomorphology of histiocytoid breast carcinoma can lead to potential misdiagnoses, differentials of which include the following entities.

\section{Histiocytic inflammatory reaction}

As its name implies, tumour cells of histiocytoid breast carcinoma resemble histiocytes with their abundant cytoplasm and relatively banal and sometimes eccentrically placed nuclei. An inflammatory histiocytic process needs to be considered in the differential diagnosis. Histiocytes can be seen in association with duct ectasia where histiocytes occupy the dilated duct lumen as well as spill out into the duct walls, ${ }^{31}$ sometimes effacing the lining epithelium that can be obscured by the inflammatory process, such that only a collection of histiocytes remain, leading to appearances reminiscent of histiocytoid breast carcinoma (figure 3A). Sometimes, foreign body type giant cells and cholesterol clefts may be seen, and the term xanthogranulomatous mastitis may be used to refer to this histological finding. ${ }^{32}$
Figure 3 (A) Histiocytes of duct ectasia, where in the absence of an intact duct, the histiocytic population can resemble the tumour cells of histiocytoid breast carcinoma. Inset shows CK7 decorating the residual epithelium of a duct that is partially effaced by histiocytes. (B) Histiocytes in post-chemotherapy changes, with a duct in the lower field containing pleomorphic cells of residual ductal carcinoma in situ.
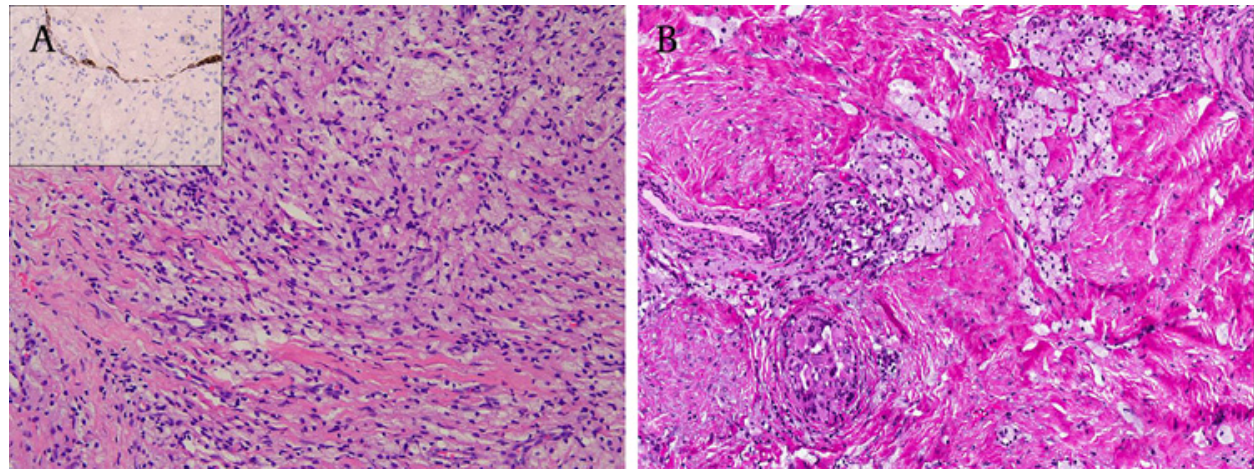


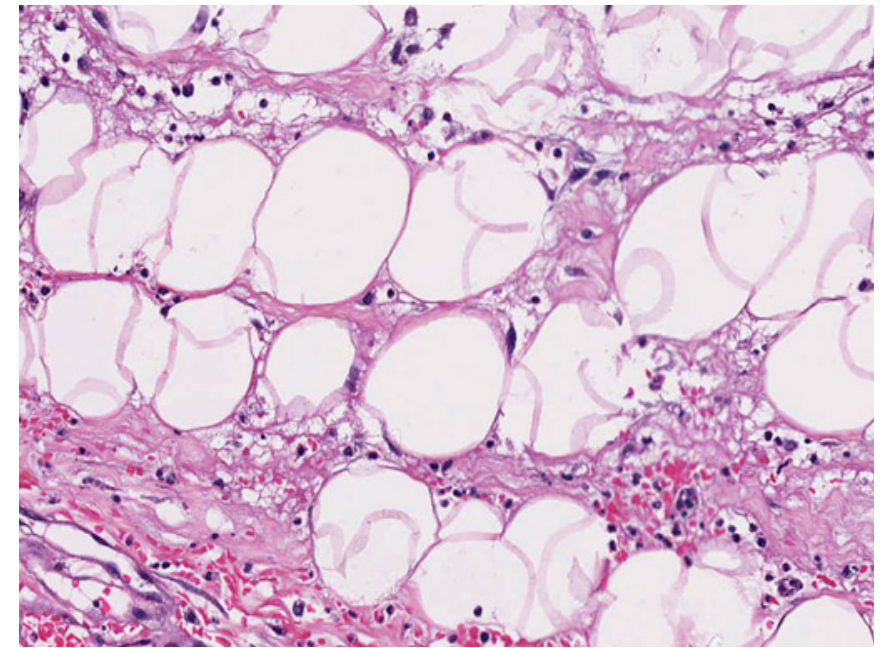

Figure 4 Fat necrosis shows necrotic adipocytes with histiocytes and other inflammatory cells.

Clues to a benign histiocytic inflammatory process are the presence of other accompanying inflammatory cells such as lymphocytes and plasma cells, and the generally limited localisation of inflammatory cells centred around a disrupted duct. Lobular neoplasia is not a usual association. Immunohistochemically, histiocytes are negative for epithelial markers and will react positively with CD68.

An exceedingly rare histiocytic process that has been described in the breast is Erdheim-Chester disease, which is a non-Langerhans cell histiocytosis of unknown aetiology that more commonly affects long bones, skin, orbit, pituitary and retroperitoneum. ${ }^{33}$ Histologically, a xanthomatous infiltrate is punctuated by Touton-type giant cells and patchy lymphocytes, which occasionally zone to a perivascular location. Immunohistochemically, CD68 decorates the histiocytes, with negative reactivity for $\mathrm{S} 100, \mathrm{CD} 1 \mathrm{a}$ and cytokeratins.

Post-neoadjuvant chemotherapy, the accumulation of foamy histiocytes may mark the location of chemotherapy-induced tumour dissipation (figure 3B).

\section{Fat necrosis}

The microscopic appearances of fat necrosis include histiocytes occurring in relation to necrotic adipocytes (figure 4). A foreign body giant cell reaction and other inflammatory cells are frequently present. Fat necrosis in the breast is often encountered in association with reactive and reparative changes in the context of a previous needling/core or surgical biopsy procedure for which the history should be readily available. However, it can also present as a spontaneous breast lump in a woman, with

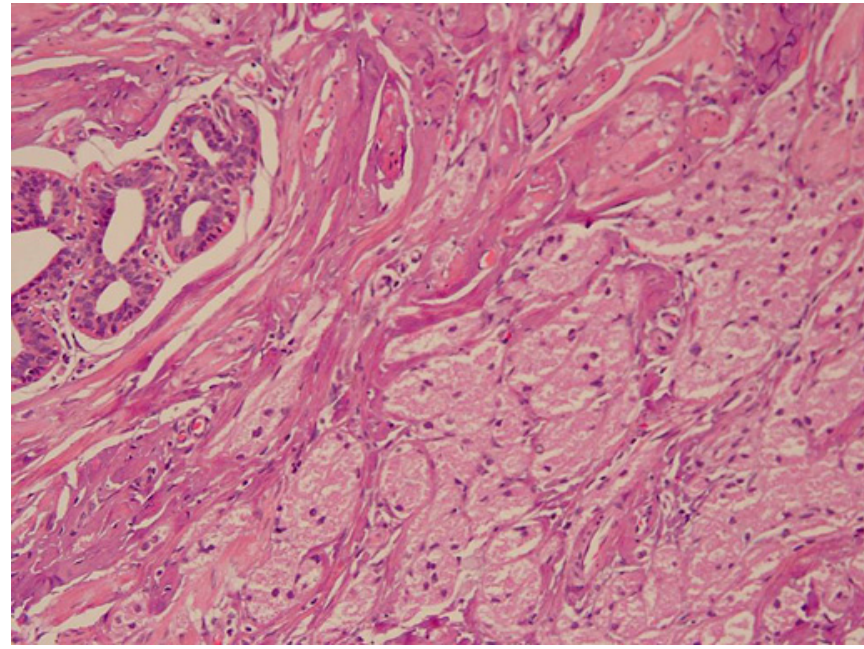

Figure 5 Granular cell tumour shows cells with abundant pink granular cytoplasm and inconspicuous cell borders.

or without a history of breast trauma. Fat necrosis can occur post-radiation treatment for breast carcinoma, ${ }^{34}$ and very rarely, lupus panniculitis has been reported in the breast of patients with systemic lupus erythematosus. ${ }^{35}$

The histological confusion of fat necrosis with a malignant process is particularly problematical during intraoperative frozen sections in which nuclear atypia of reactive histiocytes can appear accentuated. The correct diagnosis is aided by identifying accompanying inflammatory cells with reactive alterations and foreign body giant cells, as well as obtaining a proper clinical history.

\section{Granular cell tumour}

The granular cell tumour is a rare, usually benign tumour of Schwann cell derivation that can be discovered in the breast (figure 5). ${ }^{31}$ Clinically and radiologically mimicking breast malignancy, the granular cell tumour consists of sheets and nests of polygonal to occasionally spindle cells with ample cytoplasm containing eosinophilic granules that are periodic acid Schiff positive and diastase resistant. Some vacuolised and clear cells may be identified. Nuclei are vesicular with modest pleomorphism. Occasional distinct nucleoli are found. Nerve twigs may be observed in association with the granular cells.

Immunohistochemically, the cells are positive for S100 and CEA, and are negative for oestrogen and progesterone receptors. They are usually negative for histiocyte-associated antigens including $\alpha 1$-antitrypsin and $\alpha 1$-antichymotrypsin, although some reactivity for CD68 has been described.
Figure 6 (A) Rosai-Dorfman disease shows voluminous histiocytes with lymphocytes and plasma cells residing within the cytoplasm, a phenomenon referred to as emperipolesis. (B) S100 immunohistochemistry shows positive cytoplasmic reactivity of the histiocytes, accentuating the presence of lymphocytes and plasma cells within the cytoplasm.
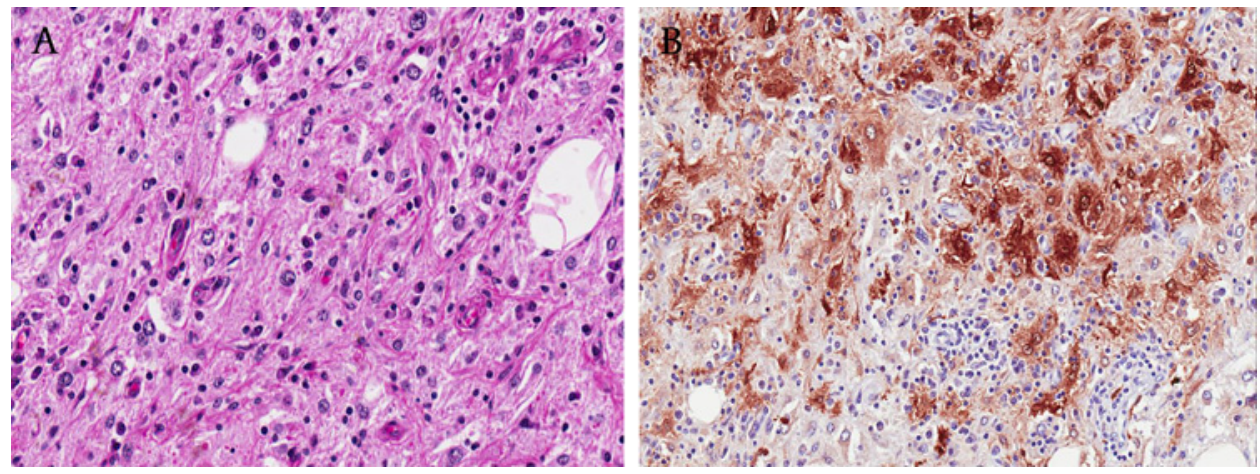


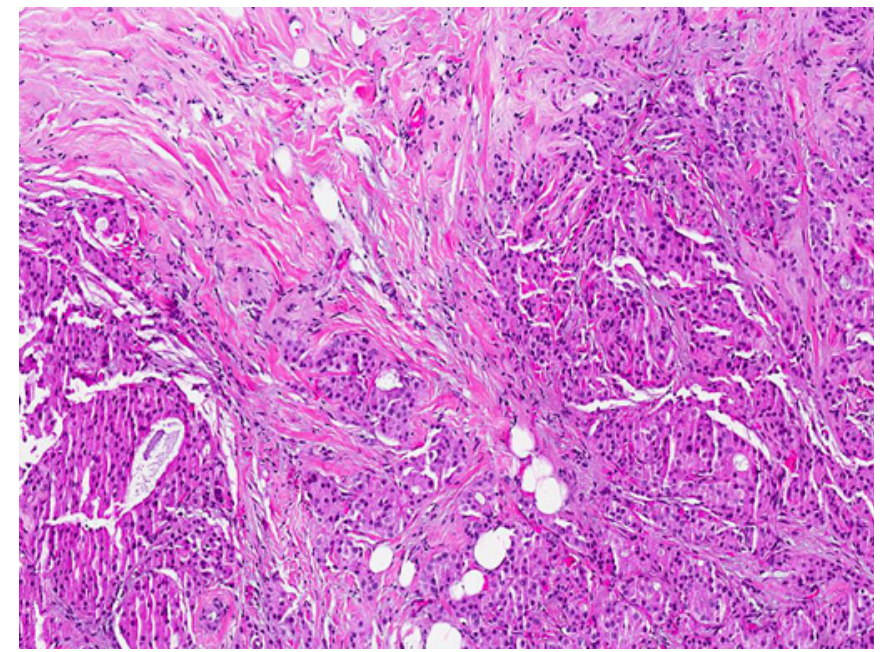

Figure 7 Invasive apocrine carcinoma with irregular nests and islands of tumour cells with apocrine appearances.

While generally benign and cured by complete excision, less than $1 \%$ of granular cell tumours are reported to be malignant. Histologically, the malignant forms display mitoses, pleomorphism, necrosis and there may be metastases, ${ }^{36}$ but instances of malignant behaviour have been observed even in the absence of these microscopic features. ${ }^{31}$

\section{Rosai-Dorfman disease}

Rosai-Dorfman disease (RDD) or sinus histiocytosis with massive lymphadenopathy (SHML) is primarily a nodal-based, idiopathic, benign proliferative disorder of histiocytes, with $43 \%$ of these cases also involving extranodal sites. The breast is an unusual site of occurrence of RDD and can clinicoradiologically mimic cancer. ${ }^{37}$

Histology of RDD consists of sheets of characteristic large histiocytes displaying emperipolesis, a microscopic hallmark of this disease (figure 6A). The predominant histiocytic population is reminiscent of the appearances of histiocytoid breast carcinoma. Lymphoid aggregates with germinal centres and plasma cells are frequent accompaniments. Immunohistochemistry shows cytoplasmic staining of histiocytes for S-100, often with weak positivity for CD68, while electron microscopy confirms histiocytic engulfment of lymphocytes and plasma cells (figure 6B).

The aetiology and pathogenesis of RDD are obscure, with an infective or immune-mediated origin being proposed. Excision is often curative. Spontaneous resolution has also been described, although a more persistent and aggressive course is possible.
RDD of the breast tends to be resected as malignancy is often clinically and radiologically suspected.

\section{Invasive apocrine carcinoma}

This histological variety is defined by the presence of apocrine cells in more than $90 \%$ of tumour cells (figure 7). ${ }^{21}$ Apocrine differentiation can be observed in any type and grade of breast carcinoma, and its recognition is of no current predictive value. Two cell types are described in apocrine cancer: type A cells, which are intensely eosinophilic and contain abundant granular cytoplasm (resembling conventional apocrine cells), and type B cells, which are foamy with ample finely vacuolated cytoplasm. The latter are histiocyte-like and would be histologically synonymous with histiocytoid breast carcinoma, affirming its apocrine immunophenotypic expression. ${ }^{3} 4$

\section{Metastasis to the breast}

Unusually, the breast can be the site of metastatic disease. ${ }^{38} 39$ Examples of metastases to the breast that can histologically display plump polygonal cells resembling histiocytoid breast carcinoma are renal cell carcinoma, melanoma (figure 8A,B) and alveolar soft part sarcoma. Correlation with clinical history and radiological findings, together with adjunctive immunohistochemistry, can lead to the correct diagnosis.

\section{CONCLUSIONS}

Histiocytoid invasive breast carcinoma is an unusual form of breast cancer, with the weight of evidence supporting its classification as a variant of invasive lobular carcinoma. Consistent GCDFP-15 expression suggests apocrine differentiation, although perhaps in a rudimentary form of cytomorphological expression. While histiocytoid breast carcinoma has been observed in relation to pleomorphic lobular carcinoma, whether its clinicobiological behaviour mirrors the aggressive characteristics of the latter variant is uncertain. Its resemblance to benign entities is a pitfall, and awareness of these mimics is needed in order to arrive at a correct diagnosis for appropriate patient management. Metastases to lymph nodes and skin can also be overlooked as benign sinus histiocytes and xanthomatous dermal lesions, respectively, ${ }^{1}$ with more disseminated spread to the uterus and parathyroid also being documented. ${ }^{40}$

Hints to the correct diagnosis include the presence of accompanying tumour cells that are more pleomorphic and mitotically active, cells with cytoplasmic vacuoles and targetoid secretions, architectural patterns resembling those of invasive lobular cancer with linear files and concentric encirclement of lobules, associated classic invasive lobular carcinoma or lobular neoplasia, and the use of adjunctive immunohistochemistry to
Figure 8 (A) Metastatic renal cell carcinoma to the breast, showing polygonal cells with ample cytoplasm and vesicular nuclei containing distinct nucleoli. Polymorphs are also seen. (B) Metastatic melanoma to the breast with sheets of plump cells adjacent to necrosis. Inset shows melanA immunohistochemical positivity.
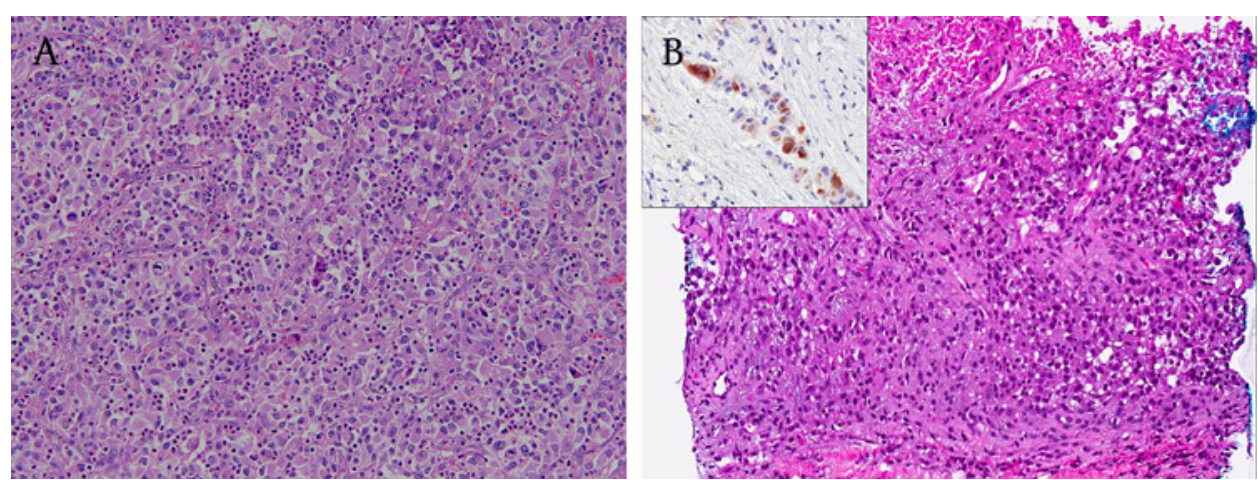


\section{Take-home messages}

- Histiocytoid breast carcinoma is an unusual tumour that is most often regarded as a variant of invasive lobular cancer.

- The bland histological appearances may mimic benign breast conditions, which pose diagnostic pitfalls especially on limited material such as core biopsies or fine needle aspiration cytology.

- Clues to the correct diagnosis are the presence of cells with more overt cytological atypia and mitoses, cells with cytoplasmic vacuoles and secretions, accompanying components of classic invasive lobular carcinoma and/or lobular neoplasia, aided by immunohistochemistry to confirm the epithelial nature of the histiocytoid cells.

- It is important for close clinicoradiological correlation, as any discordance should spur further diagnostic determination.

\section{Interactive multiple choice questions}

- This JCP article has an accompanying set of multiple choice questions (MCOs). To access the questions, click on BMJ Learning: take this module on BMJ Learning from the content box at the top right and bottom left of the online article. For more information please go to: http://jcp.bmj.com/education. Please note: the MCOs are hosted on BMJ Learning the best available learning website for medical professionals from the BMJ Group. If prompted, subscribers must sign into JCP with their journal's username and password. All users must also complete a onetime registration on BMJ Learning and subsequently log in (with a BMJ Learning username and password) on every visit.

verify the epithelial origin of lesional cells. Close clinicoradiological correlation is critical, as discordant findings on core biopsy or cytology should prompt histological pursuit of a conclusive diagnosis on open excision. In the presence of an earlier breast carcinoma, development of skin nodules comprising histiocyte-like cells should also be diligently assessed to rule out metastasis.

Competing interests None.

Contributors PHT wrote the paper, with contributions from $\mathrm{OH}, \mathrm{AAT}$ and GMKT.

Provenance and peer review Not commissioned; externally peer reviewed.

\section{REFERENCES}

1. Hood CI, Font RL, Zimmerman LE. Metastatic mammary carcinoma in the eyelid with histiocytoid appearance. Cancer 1973;31:793-800.

2. Walford N, ten Velden J. Histiocytoid breast carcinoma: an apocrine variant of lobular carcinoma. Histopathology 1989;14:515-22.

3. Eusebi V, Betts $\mathrm{C}$, Haagensen DE Jr, et al. Apocrine differentiation in lobular carcinoma of the breast: a morphologic, immunologic, and ultrastructural study. Hum Pathol 1984:15:134-40.

4. Eusebi V, Foschini MP, Bussolati G, et al. Myoblastomatoid (histiocytoid) carcinoma of the breast. A type of apocrine carcinoma. Am J Surg Pathol 1995;19:553-62.

5. Eusebi V, Magalhaes F, Azzopardi JG. Pleomorphic lobular carcinoma of the breast: an aggressive tumor showing apocrine differentiation. Hum Pathol 1992;23:655-62.

6. Fujiwara M, Horiguchi M, Mori S, et al. Histiocytoid breast carcinoma: solid variant of invasive lobular carcinoma with decreased expression of both E-cadherin and CD44 epithelial variant. Pathol Int 2005;55:353-9.

7. Kostopoulos I, Barbanis S, Mylona E, et al. Histiocytoid breast carcinoma: a case report of an uncommon histologic variant of lobular carcinoma. Ann Pathol 2003;23:249-52.
8. Cangiarella J, O'Connell Mazzei E, Weg N, et al. Aspiration biopsy in a case of apocrine adenocarcinoma with foam cells (myoblastomatoid or histiocytoid adenocarcinoma). Diagn Cytopathol 2002;26:320-3.

9. Reis-Filho JS, Fulford LG, Freeman A, et al. Pathologic quiz case: a 93-year-old woman with an enlarged and tender left breast. Histiocytoid variant of lobular breast carcinoma. Arch Pathol Lab Med 2003;127:1626-8.

10. Ramos CV, Taylor HB. Lipid-rich carcinoma of the breast. A clinicopathologic analysis of 13 examples. Cancer 1974;33:812-19.

11. Eisenberg BL, Bagnall JW, Harding CT 3rd. Histiocytoid carcinoma: a variant of breast cancer. J Surg Oncol 1986;31:271-4.

12. Hutchinson CB, Geradts J. Histiocytoid carcinoma of the male breast. Ann Diagn Pathol 2011:3:190-3.

13. Omeroglu G, Holloway CM, Spayne J, et al. Histiocytoid variant of lobular carcinoma: a triple negative case. Breast J 2010;16:84-6.

14. Murali R, Salisbury E, Pathmanathan N. Histiocytoid change in breast carcinoma: a report of 3 cases with an unusual cytomorphologic pattern of apocrine change. Acta Cytol 2006;50:548-52.

15. Kitamura H, Shimizu S, Matsukawa $H$, et al. Histiocytoid breast carcinoma: a case report with immunohistochemical and ultrastructural studies. Breast Cancer 1996:3:57-63.

16. Page DL. Special types of invasive breast cancer, with clinical implications. Am J Surg Pathol 2003;27:832-5.

17. Orvieto $\mathbf{E}$, Maiorano $\mathrm{E}$, Bottiglieri $\mathrm{L}$, et al. Clinicopathologic characteristics of invasive lobular carcinoma of the breast: results of an analysis of 530 cases from a single institution. Cancer 2008;113:1511-20.

18. Reis-Filho JS, Fulford LG, Lakhani SR, et al. Pathologic quiz case: a 62-year-old woman with a $4.5-\mathrm{cm}$ nodule in the right breast. Lipid-rich breast carcinoma. Arch Pathol Lab Med 2003;127:e396-8.

19. Kasashima S, Kawashima A, Zen Y, et al. Expression of aberrant mucins in lobular carcinoma with histiocytoid feature of the breast. Virchows Arch 2007:450:397-403.

20. Gupta D, Croitoru CM, Ayala AG, et al. E-cadherin immunohistochemical analysis of histiocytoid carcinoma of the breast. Ann Diagn Pathol 2002;6:141-7.

21. Tavassoli FA, Devilee P. World Health Organization, International Agency for Research on Cancer, International Academy of Pathology. Pathology and genetics of tumours of the breast and female genital organs. Lyon: IARC Press, 2003.

22. Shimizu S, Kitamura H, Ito $\mathrm{T}$, et al. Histiocytoid breast carcinoma: histological, immunohistochemical, ultrastructural, cytological and clinicopathological studies. Pathol Int 1998:48:549-56.

23. Da Silva L, Parry S, Reid L, et al. Aberrant expression of E-cadherin in lobular carcinomas of the breast. Am J Surg Pathol 2008;32:773-83

24. Rakha EA, Patel A, Powe DG, et al. Clinical and biological significance of E-cadherin protein expression in invasive lobular carcinoma of the breast. Am J Surg Pathol 2010;34:1472-9

25. Mossler JA, Barton TK, Brinkhous AD, et al. Apocrine differentiation in human mammary carcinoma. Cancer 1980;46:2463-71.

26. Eusebi V, Millis RR, Cattani MG, et al. Apocrine carcinoma of the breast. A morphologic and immunocytochemical study. Am J Pathol 1986;123:532-41.

27. Mazoujian G, Warhol MJ, Haagensen DE Jr. The ultrastructural localization of gross cystic disease fluid protein (GCDFP-15) in breast epithelium. Am J Pathol 1984;116:305-10.

28. Vargas AC, Lakhani SR, Simpson PT. Pleomorphic lobular carcinoma of the breast: molecular pathology and clinical impact. Future Oncol 2009:5:233-43.

29. Weigelt B, Geyer FC, Natrajan R, et al. The molecular underpinning of lobular histological growth pattern: a genome-wide transcriptomic analysis of invasive lobular carcinomas and grade- and molecular subtype-matched invasive ductal carcinomas of no special type. J Pathol 2010;220:45-57.

30. Chen YY, Hwang ES, Roy R, et al. Genetic and phenotypic characteristics of pleomorphic lobular carcinoma in situ of the breast. Am J Surg Pathol 2009;33:1683-94.

31. Rosen PP. Rosen's breast pathology. 3rd edn. Philadelphia: Wolters Kluwer Health/ Lippincott Williams \& Wilkins, 2009.

32. Koo JS, Jung W. Xanthogranulomatous mastitis: clinicopathology and pathologica implications. Pathol Int 2009:59:234-40.

33. Provenzano E, Barter SJ, Wright PA, et al. Erdheim-Chester disease presenting as bilateral clinically malignant breast masses. Am J Surg Pathol 2010;34:584-8.

34. Coyne JD, Parkinson D, Baildam AD. Membranous fat necrosis of the breast. Histopathology 1996;28:61-4.

35. Sabate JM, Gomez A, Torrubia S, et al. Lupus panniculitis involving the breast. Eur Radiol 2006;16:53-6.

36. Chetty R, Kalan MR. Malignant granular cell tumor of the breast. J Surg Oncol 1992; 49:135-7.

37. Ng SB, Tan LH, Tan PH. Rosai-Dorfman disease of the breast: a mimic of breast malignancy. Pathology 2000;32:10-15.

38. Chia SY, Thike AA, Cheok PY, et al. Utility of mammaglobin and gross cystic disease fluid protein-15 (GCDFP-15) in confirming a breast origin for recurrent tumors. Breast 2010:19:355-9.

39. Lee AH. The histological diagnosis of metastases to the breast from extramammary malignancies. J Clin Pathol 2007:60:1333-41.

40. Allenby PA, Chowdhury LN. Histiocytic appearance of metastatic lobular breast carcinoma. Arch Pathol Lab Med 1986;110:759-60. 